\title{
The dynamical fragile-to-strong crossover in attractive colloidal systems
}

\author{
Francesco Mallamace ${ }^{\text {tr }}, 1,2$, Carmelo Corsaro ${ }^{1}$, Cirino Vasi $^{3}$, Sebastiano Vasi ${ }^{3}$, \\ Domenico Mallamace ${ }^{4}$ and Sow-Hsin Chen ${ }^{2}$. \\ ${ }^{1}$ Dipartimento di Fisica e Scienze della Terra, Università di Messina I-98168 Messina, \\ Italy. ${ }^{2}$ Department of Nuclear Science and Engineering, Massachusetts Institute of \\ Technology, Cambridge MA 02139. ${ }^{3}$ CNR-IPCF Istituto per i Processi Chimico-Fisici, \\ I-98158 Messina, Italy. ${ }^{4}$ Dipartimento SASTAS Università di Messina, I-98168 Messina, \\ Italy.
}

\begin{abstract}
The dynamics of a hierarchical colloid solution, dendrimer in methanol, has been studied using photon-correlation spectroscopy (PCS). The obtained relaxation curves are analyzed according to the mode-coupling theory (MCT). The main results are: i) the occurrence of a fragile to strong dynamical crossover as a function of the packing fraction at $\phi_{c} \simeq 0.39$, and ii) the observation of a secondary relaxation $(\beta)$ that has its onset just at the dynamical crossover and is correlated with the primary $\alpha$-relaxation. Finally we discuss the obtained findings in comparison with recent experiments in similar systems characterized by an attractive interaction obtaining a confirmation on the primary role of the dynamical crossover in the physics of the dynamical arrest.

Keywords: Diverging processes, Dynamic Crossover, Fragile to strong transition, Glass forming materials, Soft-matter
\end{abstract}

\section{Introduction.}

The dynamic arrest phenomenon (DA), accompanied by a remarkable dynamic slowing down that marks a dramatic change in the properties of a physical system [1], represents an hot topic in condensed matter research. The

\footnotetext{
Corresponding author

Email address: francesco.mallamace@unime.it

Preprint submitted to Journal of Non-Crystalline Solids

July 16, 2014 
5 phenomenon is customarily studied by exploring the transport coefficients (e.g., viscosity $\eta$, self-diffusion constant $D_{s}$, and relaxation time $\tau$ ) as functions of thermodynamical variables (temperature $T$ and concentration $C$ ). The dependence of the supercooled liquids dynamics into a metastable state below their melting temperature $T_{M}$ is well known. The study of the way of their approaching to these limiting values may provide information about the nature of the process. A special situation regards these coefficients, and $\tau$ in particular that, as $C$ increases or $T$ decreases below $T_{M}$, can change by several orders of magnitude, eventually surpassing the time required for experimental accessibility. In some cases, this behaves, as proposed by the ideal mode-coupling theory (IMCT) [2], the breaking of the system's ergodicity in the amorphous glass phase at or below the glass transition temperature $T_{g}$. This approach has received much attention in the glass-transition literature, but the usual methodology is to explore the $T$-dependence of these coefficients by supercooling liquids into the metastable state below the melting temperature $T_{M}$ until they either crystallize or vitrify. Often, the packing fraction (or the volume fraction $\phi$ ) can be the control parameter $[2,3,4,5,6,7]$, e.g., in complex fluids like polymers and colloids.

Considering the various open questions on the related basic processes, the DA constitutes an exciting and challenging research topic today while strongly 25 debated also with conflicting opinions $[8,9,10,11,12,13,14]$. One of these deals with a possible "DA criticality": i.e., whether transport parameters reflect an underlying phase transition to a state in which these quantities diverge at a finite temperature (the "dynamic divergence"). Another one instead focuses on the occurrence, or not, of the system non-ergodicity inside the glass phase originally observed in hard colloidal solutions and explained according to the MCT theory $[4,5]$. For this latter argument it has been recently proposed that grafted polydisperse colloids [15] and molecular liquids with internal degrees of freedom remain ergodic at all temperatures $[9,16]$ and therefore statistical mechanics can be applied to describe the arrest process.

Transport coefficients on approaching DA, have been treated by means of 
the empirical Vogel-Fulcher-Tammann (VFT or super Arrhenius) form: $\tau=$ $\tau_{0} \exp \left(B T_{0} /\left(T-T_{0}\right)\right)$. The significant reason of its extensive use is in the indication of a finite diverging temperature $T_{0} \ll T_{g}$, associated with the Kauzmann temperature $T_{K}[17,8]$ and for the liquids classification in two separate categories: "fragile" or "strong" glass formers [18]. Whereas "strong" liquids exhibit pure Arrhenius $T$-dependence $: \ln \left(\tau / \tau_{0}\right)=E / k_{B} T$, the "fragile" ones have a marked VFT behavior. Despite of its empiricism, VFT has been treated as a "universal" feature and only recently its validity has been reconsidered because different experimental studies and theoretical reasoning show that it lacks of a true physical meaning $[9,19,11,12,20]$.

Various recent studies $[10,12,20]$ suggest that for the study of liquid dynamics in a broad $T$-range, at least two dynamic regimes are necessary; the reason lies in an intriguing phenomenon occurring at a temperature $\left(T_{B}\right)$ well above $T_{g}$ : the so called fragile to strong dynamic crossover (FSDC). Lately, $T_{B}$, for the processes disclosing their properties there, has been recognized as a datum point in the DA being the FSDC accompanied by several significant phenomena like: (i) the broadening of the structural relaxation time distribution accompanied in some cases by the loss of ergodicity, conditions for which the scattering functions $F(q, t)$ can be described by means of a stretched exponential ${ }_{55} F(q, t)=F_{q}^{c} \exp \left[-\left(t / \tau_{\alpha}\right)^{\beta}\right]$ and can approach an almost constant value for long times $[4,5]$; (ii) the splitting of the high- $T$ relaxation into the primary $(\alpha)$ and the Johari-Goldstein $\beta_{J G}$ relaxation times (also named secondary or $\beta_{\text {slow }}$ ) [21]; (iii) the decoupling between the translational and the rotational diffusion (for $T<T_{B}$ ) corresponding to a violation of the Stokes-Einstein (SE) relation. In the high $T$-regime, as evidenced by the $\mathrm{SE}$, the translational diffusion, $D_{s}$, tracks the inverse of the shear viscosity $\left(\eta^{-1}\right)$ whereas for $T<T_{B} D_{s}$ declines far less rapidly by decreasing $T$ as $D_{s} \sim \eta^{-\xi}$, with $\xi \simeq 0.75$; instead $D_{R o t}$ (or the rotational correlation time) remains proportional to the inverse of the shear viscosity down to $T_{g}[22,23,24,25]$. For this reason any experimental technique sensitive only to the $D_{\text {Rot }}$ cannot observe the violation; (iv) for a broad set of supercooled systems $\tau\left(T_{B}\right)=10^{-7 \pm 1} \mathrm{sec}$ and $\eta\left(T_{B}\right) \approx 10^{3} \mathrm{~Pa}$ sec are near-universal 
[20] and if we consider the $T-P$ phase diagram: $\tau\left(T_{B}, P_{B}\right), \eta\left(T_{B}, P_{B}\right)=$ const for a given glass former; (v) it is believed that the dynamical crossover is closely related to the appearing of dynamical heterogeneities $(\mathrm{DH})$, although some pure Arrhenius systems show them [26]. DH refers to the presence of transient spatially separated regions with vastly different relaxation times [22, 23, 27]. (vi) usually a fragile to less fragile (or strong) transformation occurs when passing $T_{B}[20]$; (vii) and finally the hopping extended MCT (EMCT) identifies $T_{B}$ with the MCT critical temperature $T_{c}[2,28,29] . T_{B}$ can be evaluated by means of different methods, such as by means of a plot based on the $T$-derivative of the $\alpha$-relaxation time [30].

Exploration of all these phenomena can help to stress more the importance of the dynamical crossover [10, 31, 32, 33, 34], and its universality [20].

Must be noticed that although the FSDC is a datum poin for the dynamical arrest, in the case of hydrated proteins the FSDC represents a debated subject with controversial opinions [35, 36, 37, 38]. In this latter case it has been proposed that the FSDC may be related to the splitting of a secondary relaxation [36]. Whereas for glass former the transition is related to the molecular cage dynamics [29] for hydrated proteins its origin appears unclear. However, a comparative analysis of the dynamical properties of proteins hydration water as far as bulk and confined water coming from many different experimental techniques and proper molecular dynamic simulations (MD), reveals that all the reported data are consistent with the dynamical crossover scenario [37].

Different approaches for estimating $T_{B}$ have been recently proposed: one is based on the MCT [20, 29], the second employs the constraints of the AdamGibbs theory $[12,31]$ and the most recent one explores the apparent enthalpy space properties [10]. All of them stress the basic importance of the dynamical crossover in the DA physics. The dynamic crossover concept can be considered of true interest not only for the way in which a system arrests its dynamics but also for the new science frontiers towards the mesoscales. In fact very recently 
the crossover has been explicitly considered as a way with which multiscale materials can model the mesoscale, i.e. how the understanding of mechanisms at the microscale can enable predictions of functional behavior at the macroscale [31]. For these reasons $T_{B}$ appears to be of central interest in material science.

We also stress that by considering the relevant results in the field of the dynamical crossover its dependence on the concentration can be an useful test of some important suggestions such as the $\phi$-dependence of the $\beta$-secondary relaxation strength could be useful to understand if this process has its onset just at the FSDC. Furthermore, on this respect it is noteworthy to verify how the "onset" scenario of this $\beta$-process can be related with the crossover scenario for the JohariGoldstein $\left(\beta_{\mathrm{JG}}\right)$ relaxation and $\gamma$-secondary relaxations observed in sured correlators are studied in the current MCT frame with the following main objectives: a) to obtain a careful verification of the dynamical crossover in the primary $\alpha$-relaxation; b) to explore whether the system maintains the ergodicity 
near and below the glass transition, and whether the dynamical crossover exists with this property; c) to accurately compare the obtained data with the related findings recently observed in other colloidal systems [15].

Experiment and Results.

It is well known that in the QELS experiment the field autocorrelation function, $S_{1}(q, t)$, related with the translational and diffusional molecular modes is measured [39]. Just to have only a measure of the density correlations, we worked in a polarized geometry $(V V)$ so that the present PCS experiment measures the correlation function, $g_{1}(q, t)$.

The colloidal solution we studied is a polyamidoamine (PAMAM, with ethilenediamine central core) dendrimer (or starbust polymers) of generation six ( $g$ $140=6$ ) in methanol. These macromolecules, synthesized by repeating units in a hierarchical self-similar fashion, have a relatively homogeneous radial density with flexible end groups (with a certain degree of backfolding). More precisely, whereas, the low generations $g<5$ have a fractal like internal structure, for generations $g \geq 5$, the scattering distributions are consistent with an homogeneous sphere distribution monodisperse in size [40], a situation confirmed by MD simulation [41]; in addition, they can be classified as monodisperse spherical colloids. Depending on the generation number, their radius ranges from 5 to $60 \AA$, the molecular weight from some tens to $10^{6}$ Daltons (for $g=6$, $M_{W} \sim 40.000 D a$, and $R_{g} \sim 22 \AA$, the same order of magnitude of lysozyme and other small proteins). For moderate and large integer generation, $g>5$, they can be classified as true colloids having a structure characterized by an hard core plus a corona region made of the flexible end groups (whose fractional depth decreases on increasing $g$ ). In principle, these flexible end groups of the dendrimer corona can behave like the polymers of the grafted colloids (or the hydrated corona of copolymer micelles) giving rise to an attractive interparticle interaction [40].

We have used only PAMAM dendrimers of generation $g=6$, with $\mathrm{NH}_{2}$ and $O H$ terminal groups, obtained from "Dendritech Inc" as methanol solution at the weight concentration of about $10 \%$. The solvent was completely 

measure of the solute volume, sample was prepared at the desired volume fraction by adding the solvent. The studied volume fractions, $\phi$, covers a range from 0.1 to 0.61 . PCS measurements were made at a scattering angle $\vartheta_{s}=90^{\circ}$ $\left(|q|=4 \pi n / \lambda_{0} \operatorname{sen}\left(\vartheta_{s} / 2\right)\right.$ being $n$ the system refractive index and $\lambda_{0}$ the vacuum erating at $50 \mathrm{~mW}(5120 \AA)$ and an optical scattering cell of a diameter $1 \mathrm{~cm}$ in a refractive index matching bath. The sample is thermostated with a stability within $\pm 10 \mathrm{mK}$. The intensity data were also corrected for turbidity and multiple scattering effects [39]. PCS data have been taken using a digital correlator with a logarithmic sampling time scale which allows an accurate description of both the short- and long- time regions of the intermediate scattering function form $0.1 \mu \mathrm{sec}$ to $100 \mathrm{sec}$.

The system works in the homodyne mode and measures, at a given wavevector $q$, the intensity correlation function $g_{2}(q, t)=\langle I(q, 0) I(q, t)\rangle /\langle I\rangle^{2}$, related to the scattered electric field normalized autocorrelation function $g_{1}(q, t)=$ $S(q, t) / S(q, o)=\left\langle E_{s}^{*}(q, 0) E_{s}(q, t)\right\rangle /\left\langle\left|E_{s}\right|^{2}\right\rangle$ through the Siegert's relation $g_{2}(q, t)=$ $1+\left|g_{1}(q, t)\right|^{2}$. To be precise, in order to measure the normalized intensity correlation function we used a method with a special average procedure [5]

$$
g^{(2)}(q, t)=\left\langle\langle I(q, 0) I(q, t\rangle\rangle /\langle\langle I(q, 0)\rangle\rangle^{2}\right.
$$

The first bracket denotes the time average and the second bracket the positional average over different parts of the sample. The system, in fact, can show a structural arrest, and therefore a non-ergodic behavior, when the correlation functions tend to develop very long time delays. In such a situation a particular care must be taken in averaging over many different sample positions for a correct measure of the long time region of the time correlation function. For each concentration a large number of measurements must be performed, enough for a correct average, observing a larger scattering area corresponding to three or more independent Fourier components and changing the position of the sample in order to observe different scattering volumes. By using such 
a procedure, that explores many scattering volumes, for each autocorrelation function a typical overall experimental time of about 4 hours is necessary in the highest volume fraction regimes. However, for $\phi>0.3$ we have repeated several times the measuring procedure.

Figure 2 illustrates some measured $g_{1}(q, t)$ in the range from $\phi=0.15$ to $\phi=0.615$ at $293 K$. All the reported $g_{1}(q, t)$ curves are characterized by a complex relaxation and the decay to zero in finite times. Besides a very short time contribution, that is well evident for $\phi=0.15,0.27$, and identified as the cage relaxation time $\tau_{\beta f a s t}$, the correlators are characterized by the presence of other two contributions: an intermediate that appears only for $\phi \geq 0.37$ and a long-time tail (the $\alpha$-decay). Important observations are that the system ergodicity is fully preserved at all the studied concentrations and the presence of the intermediate relaxation that should be the Johari-Goldstein $\tau_{\beta J G}$ relaxation time [21].

In the region $0.11<\phi<0.37$ the $g_{1}(q, t)$ curves have been analyzed (continuous lines in Figure 2 represent the data fitting) as the superposition of an exponential short-time decay (the $\beta$ - fast contribution) and a long-time stretched tail (the $\alpha$-decay), weighted for the non-ergodicity factor $f_{c}$. As a result we find that whereas the $\tau_{\beta \text { fast }}$ results nearly insensitive by increasing $\phi$ (remains nearly constant, about $0.310^{-6} \mathrm{sec}$ ) $\tau_{\alpha}$ increases of about four orders of magnitude. For $\phi>0.37$ in addition to these two mentioned contributions, an intermediate relaxation of the order of some $m$ sec appears. Being these three contributions separated by about three orders of magnitude each, we have used, in order to process the data, together with the previous two exponential forms, another stretched exponential. Hence from the data fitting, we obtain the times $\tau_{\alpha}, \tau_{\beta f a s t}$, and $\tau_{\beta i n t e r}$, the two stretched exponents $\beta, \beta_{\text {inter. }}$ and finally the non-ergodicity factor $f_{c}$.

\section{Discussion.}

The ideal MCT proposes the existence of a precursor phenomenon characterized by anomalous dynamics, around the critical value of the control parameter $T_{c}$ or $\phi_{c}$, at the transition from liquid to amorphous solid well prior the 
glass transition. Inside this phase the theory interprets experimental data with closed equations of motion obtaining correlators (the density-fluctuation correlation functions $F(q, t)$ at a given wave-vector $q[2]$ ). In addition, $T_{c}$ (or $\phi_{c}$ ) is explained as a bifurcation singularity in the $F(q, t)$ long- $t$ limit, a reason for which the observables behave differently above and below it; this limit, called glass form factor (or non-ergodicity parameter $f_{c}$ ), is zero in the liquid phase but positive for the ideal glass. In the case of loss of the ergodicity, $f_{c}$ behaves discontinuously, with a diverging behavior in transport (the MCT divergence) if the control parameter passes its critical value. Thus, $f_{c}$ exhibits a singularity as a function of the distance $\epsilon=\left(T-T_{c}\right) / T_{c}\left(\right.$ or $\left.\epsilon=\left(\phi-\phi_{c}\right) / \phi_{c}\right)$ for $\epsilon=0$. Using $\phi$ as the control parameter, hard (HS) or soft (SS) spheres (like 225 colloidal particles $[4,5,15])$ have been largely used to study the DA by means of the MCT. These systems, at thermal equilibrium have large viscosity (and viscoelastic) and can form an amorphous solid [42] at large $\phi$. We note that the HS system has been used as a theoretical paradigm for a simple fluid and that experiments conducted on HS colloids indicate that it can also serve as an accurate test of MCT $[4,5]$. Originally it appeared that the ideal glass transition concentration of the MCT was identical to the "calorimetric" glass transition, $\phi_{c}=\phi_{g}$. This suggests that studies of colloidal systems can help us understand the conventional glass transition, which is usually studied in molecular liquids.

MCT dynamics is based on the molecular caging for which the correlator behavior is described according to precise scaling laws (also supporting the arrest universality). Below $T_{c}$ the interactions of density fluctuations arrest the previous region via cage-effects and the molecular origin of the system dynamic evolution represents an open question. The MCT scaling form commonly used to analyze the transport parameters, and to calculate $\phi_{c}$ inside the arrest region, is in the $\tau_{\alpha}(\phi)$ case:

$$
\tau_{\alpha}=\tau_{\alpha}(\epsilon)=\tau_{\alpha 0}\left[\frac{\phi-\phi_{c}}{\phi_{c}}\right]^{-\gamma},
$$

where $\gamma$ is a non-universal exponent, the same law holds for T. $\phi_{c} \approx 0.57-$ 2350.59 are the most accepted values for the location of the colloidal glass transition; 
it has been also widely believed that a truly non-ergodic state is obtained at larger $\phi$. Eq. 3 describes well transport data of fluids in the region $\phi<\phi_{c}$, and thus in the region from the liquid stable phase to the coupled fluid phase. In the same region MCT characterizes the measured structural relaxations as the bimodal decay (primary $\alpha$ - and secondary $\beta$-processes) in $F(q, t)$ observing the way in which molecules explore all the available cage space. In the quasi-elastic scattering (QES) time regime, the two contributions are superimposed, with the $\beta$ contribution at the lowest time $\left(\beta_{\text {fast }}\right)$. The IMCT proposes precise scaling laws and exponents for both these relaxations. The ideal MCT divergence is represented by these scaling law forms: designating $\sigma=C \epsilon$ as the separation parameter, the $\beta$-relaxation is described by $t_{\sigma}=t_{0} /|\sigma|^{1 / 2 a}(1 / 2 a>1.27)$. In the $\alpha$ - scaling law regime, the IMCT gives another power-law divergence (the $\gamma$ exponent is larger than that of $t_{\sigma}(1 / 2 a)$, i.e., $\tau_{\alpha}=B^{-1 / b} t_{0}|\sigma|^{-\gamma}$ and $\gamma=[1 /(2 a)+1 /(2 b)])$. The critical exponents $0<a \leq a_{\max }=0.395$ and $0<b \leq 1$ are related through the system-dependent parameter $\lambda(1 / 2 \leq \lambda<1)$ as: $\lambda=\Gamma(1-a)^{2} / \Gamma(1-2 a)=\Gamma(1+b)^{2} / \Gamma(1+2 b)$. QES experiments made in hard-sphere colloids for $\phi<\phi_{c}$, which measure both the self-diffusion coefficient $D_{s}$ and $\tau_{\alpha}$, fully support these suggestions, giving $\gamma=2.7$ [5]. In polystyrenenetworked-sphere colloids, however, $\gamma=3.6$ and $\lambda=0.88$ [43].

The MCT has been intensively studied by means of experimental and simulation approaches in molecular systems and colloids. There is however a difference in these systems: for the same molecular glass formers the transition from an ideal MCT regime to an activated one has been experimentally demonstrated. Hence, it was possible to consider proper extended MCT models [2]. For colloidal hard spheres the situation remains essentially unclear for the limited range of the corresponding dynamical data. If compared with the 15 orders of magnitude for which the molecular fluids dynamic data are measurable, for colloids, depending on the particle size, is in fact available a much smaller range, typically eight decades or less. To be precise, one must note the experimental time window available for these two classes of systems; whereas in the case of molecular materials the $\alpha$-relaxation can be explored in a time relaxation interval ranging 
from $10^{-13}$ to $10^{2} \mathrm{sec}$, for colloidal systems this range can be actually, by means of a proper use of the dynamical light scattering technique (DLS) [15], $10^{-4}$ to $10^{4} \mathrm{sec}$. In the past by means of traditional DLS the range for colloids was very limited: $10^{-4}$ to $1 \mathrm{sec}$ [5], whereas here (see Figure 2) we have $10^{-7}$ to $100 \mathrm{sec}$.

A special feature of the EMCT is the prediction of a dynamic crossover in the transport parameters occurring just near the critical values of the control parameter associated to the dynamic change in the system, from one determined by the cage effect to one characterized by the hopping processes. It is a FS crossover in which the $\alpha$-relaxation time and the self-diffusion undergo a crossover from SA to AE behavior. This result, obtained for a Lennard-Jones system, explains the FS crossover observed in a variety of glass-forming fluids $[28,44]$. A temperature that marks dynamical changes of fragile supercooled liquids below $T_{M}$, has been already considered $[11,12,45,46]$ and from these suggestions started the idea that $T_{c} \equiv T_{B}[20,29,10]$.

EMCT explains the scale decoupling by assuming two possible diffusional contributions: i.e. a very long time thermally activated hopping process $\left(D^{h o p}\right)$ and the one typical of the IMCT $\left(D^{i d}\right)$; hence the total self-diffusion coefficient is $D_{s} \approx D^{h o p}+D^{i d}$, characterized by the dynamical crossover at $\phi_{c}$ (or $T_{c}$ ) from $D \approx D^{i d}$ to $D^{h o p}$. $D^{i d}$, characterizing the kinetic glass transition (the Super-Arrhenius step of the DA) must vanish at $T_{c} / T=1$ with a power law $D^{i d} \sim\left|T-T_{c}\right|^{\gamma}$ just reflecting the predictions by the idealized theory. Hence, the dynamical behavior of the system towards the arrest at $T_{g}$ is dominated by the hopping (Arrhenius) process with only one energy barrier (the second DA 290 step).

The IMCT power law fits the transport parameter data in the SA region and indicates the control parameter value at which the experimental data cross that of the Arrhenius behavior. This analysis indicates the physical significance of the crossover concept in terms of a well-established theory of glass-forming systems, rather than of numerous empirical observations [29].

The FS crossover with $\phi$ and $T$ as control parameters has been accounted by using copolymer micellar system with short-range attraction intermicellar inter- 
action. The system is an aqueous solution of a nonionic, three-block copolymer made of polyethylene and polypropylene oxides (Pluronic L64). This polymer in water is a $T$-dependent surfactant that forms monodisperse spherical micelles in a wide $T-\phi$ range. The system is widely described in many experiments $[47,42,48,49,50,6,51,7]$, and the Figure 1 illustrates the obtained main findings. In this system the attractive interaction gives rise to a complex $(T-\phi)$ phase diagram characterized by the existence of a percolation threshold (PT) line. In this system the super-Arrhenius behavior (described using IMCT) goes from the disordered liquid phase up to the PT line, whereas a strong glassforming behavior is observable at the highest $T$ and $\phi$ values going from the PT line (that is also the locus of the $T_{c}$ and $\phi_{c}$ values) to the glass transition line. Due to the attractive interaction, and the clustering process characterizing this system, these critical values are strongly correlated in the sense that at an higher critical temperature value corresponds a lower critical volume fraction value (and viceversa).

Before discussing the relaxation times we measured, we consider, in Figure 3, the obtained non-ergodicity factor $f_{c}$ (inset) and the stretched exponent $\beta$. with the theory. It is evident from Figure 3 that $\beta_{\text {inter. }}$ (closed symbols), the streched exponent of the intermediate relaxiation is insensitive to the packing fraction variation with the value, within the experimental error, of $\beta_{\text {inter. }} \approx 0.98$ indicating that the corresponding relaxation has practically a pure exponential behavior; on the contrary the $\beta$ value (open symbols) corresponding to the longtime $\alpha$-relaxation appears to be strongly $\phi$-dependent. As can be observed, $\beta$ has different behaviors above and below $\phi \approx 0.39$, indicating such a values as the $\mathrm{MCT}$ crossover $\phi_{c}$; more precisely, besides the lowest concentration (about 1 ) for $\phi<0.39 \beta \simeq 0.7$ whereas in the range $0.39<\phi<0.5$ we have $\beta \simeq 0.5$ after that $325 \beta$ increases, evolving toward the unity at the highest $\phi$. This behavior appears to be consistent with the MCT time-temperature superposition principle [2], no $\phi$ dependence in this stretching parameter must be observed below the critical value of the control parameter $\phi_{c}$. Above this interval, where $\beta$ has a constant 
behavior, the corresponding stretched exponents are instead characterized by a marked increase towards $\beta \simeq 1$, indicating the recovering of a single exponential temporal behavior. It seems that the relaxation time decays also have to obey to the same precise two-stage activation process indicated by the strong Arrhenius behavior of transport parameters, in a way fully consistent with the hopping process of the EMCT. The $f_{c}$ behavior for different values of $\phi<\phi_{c}$ is represented in the range $0.4<\phi<0.61$. We found that $f_{c}$ has a value around to 0.45 and appears to be $\phi$-dependent; a behavior also observed in literature [2], in fact the MCT predicts this parameter, as $\phi$ increases, a behavior according to $\left|\phi_{c}-\phi\right|^{1 / 2}$. The data reported in the figure appear to agree with such scaling law (dashed line) although the data experimental error is fairly large in order to draw a definitive conclusion on this respect. However, this behavior agrees with the observations on a "strong" molecular glass forming system [52].

Figure 4 reports the relaxation times obtained in our experiments: $\tau_{\alpha}$, $\tau_{\beta f a s t}$, and $\tau_{\beta}=\tau_{\beta i n t e r .}$. Also these data confirm that the bifurcation from the $\alpha$ process appears close to $\phi_{c} \approx 0.39$ (with a value of $\sim 0.2 \mathrm{sec}$ ). In the same region $\tau_{\beta}$ seems to have its onset showing an Arrhenius dependence that persists also inside the glass state. The experimental points of the cage relaxation time $\tau_{\beta f a s t}$, are also reported, and as it can be observed are, on the contrary of the others, nearly $\phi$ independent. The onset of $\tau_{\beta}$ for $\phi \geq \phi_{c}$ suggests that this relaxation time, may represents just the Johari Goldstein relaxation (JGR). This relaxation is basically a molecular process that is supposed to be universal, it seems to be strongly connected to the structural $\alpha$-relaxation in all glass formers [53]. There is an experimental evidence on its connections in thermodynamic properties to the $\alpha$-process, as proved by means of the NMR spectroscopy, where it is shown how, in glass forming materials above the critical value of the control parameters, these two relaxations are correlated [54].

Usually the JGR is observed in Dielectric relaxation spectra as a peak, or as an excess wing on the high frequency flank of the primary relaxation when not resolved [53]; although some small molecular glass formers do not evidence such a phenomenon. The instrumental resolution or the thermodynamical conditions 
1 explains why the observed secondary relaxation may be the JGR; as defined, the function $g_{1}(q, t)$ measures the dynamical correlations in both orientation and position between all pairs of molecules as due to the interaction processes. Whereas the corresponding translational mutual diffusion is reflected in the The $g_{1}(q, t)$ contains all the possible molecular dynamics that, depending on the probe used, can be (or not) separately measured.

All the PCS data here reported for attractive dendrimers stress the significant role in the dynamical arrest of the dynamical crossover, in agreement with MCT glass transition concentration $\left(\phi_{c}\right)$ [15]. It is just this last study that gave the first evidence on the preservation of the system ergodicity for $\phi>\phi_{c}$ ) as shown by our correlation functions (Figure 2). The idea that molecular glass formers fluids can be characterized by a cutoff mechanism suppressing the and Brownian $[56,57]$. The main suggestion is that the same behavior must hold true for molecular and colloidal systems, whose dynamics takes place in the two-steps above described. However, from these studies emerge that the kinetic glass transition does not coincide with the ergodicity-breaking and the ergodregion.

On this subject we have to stress the difference with the findings of previous experiments on hard colloids characterized by an evident non-ergodicity [5]. A difference that can have two explanations: the first one is that the hard sphere 390 of a field-theory approach for which the transition from an IMCT regime to an activated one has been demonstrated.

In recent years the viewpoint that colloids posses the property of the MCT divergence suppressions, has been a subject of special interest and has been widely discussed especially for systems with different statistics: stochastic, Newtonian icity is maintained by varying the concentration, also in the activated hopping

system is characterized by only a pure repulsive interaction, whereas the grafted 
colloid has an interaction with an additional attractive tail; and the second is the difference in the polydispersity. On this issue some discussions $[5,58,15]$ must be considered.

In our experiment we have observed the JGR that has its onset at the dynamical crossover $\phi_{c}$. We also have high-lighted that, in the frame of the present scattering experiment, the primary, $\tau_{\alpha}$, and this latter, $\tau_{\beta J G}$, are dynamically correlated for the coupling between the rotational and translational motion of the macromolecules. We deal with attractive colloids for which an increase in the packing fraction leads to a clustering process reflected in the measured correlators not only in a pure translational dynamics. From the theoretical point of view, just in the MCT terms, $\phi_{c}$ separates two dynamical behaviors before and after it. In the first case the measured $\tau_{\alpha}$ has to obey the MCT scaling law and in the second one a concentration activated process takes place. After the critical divergence at $\phi_{c}$, a further compression imposes the crossover to another dynamical stage. Just this dynamical change towards a two energy level activated thermodynamics can be related with the JGR. In fact the JG relaxation, like the primary $\alpha$-relaxation, involves transitions between different energy basins. Only in these terms the closer connection between these relaxations and the phenomenon of dynamic heterogeneity should be possible [60]. ${ }_{410}$ The interbasin transitions for $\phi>\phi_{c}$ is also accounted by the measured values for the stretched exponents related with the two relaxations $\tau_{\alpha}$, and $\tau_{\beta J G}$. As it can be observed in the Figure 3 whereas all the measured JGR stretched exponent values are about 1 , the corresponding value of the $\alpha$-relaxation increases by increasing $\phi$ above $\phi_{c}$ until the same value.

We conclude observing that our dendrimer system behaves like a grafted colloid [15] suggesting that in the EMCT frame molecular and colloidal systems behave at the same way. From our experiment we also observe in addition with the primary $\alpha$-relaxation another one that, according to the current models, can represent the Johary Goldstein relaxation. These results give additional supports on the primary importance of the dynamical crossover in the DA.

Acknowledgments - Research at MIT was supported by the Office of Basic 
Energy Sciences, U. S. Department of Energy under contract No. DE-FG02-

90ER45429. CC thanks the Centro Siciliano di Fisica Nucleare e di Struttura della Materia (CSFNSM) for its support.

\section{References}

[1] P.G. Debenedetti. Metastable Liquids: Concepts and Principles(Princeton Univ. Press, Princeton, 1996).

[2] W. Götze. Complex Dynamics of Glass-Forming Liquids A mode-coupling theory (Oxford Univ. Press, Oxford, 2009).

[3] U. Bengtzelius, W. Götze, and A. Sjölander, J. Phys. C 17, 5915 (1984).

[4] P.N. Pusey, W. van Mengen, Nature 320 (1986) 340-342.

[5] W. van Mengen, et al., Phys. Rev. E 58 (1998) 6073-6085.

[6] S.-H. Chen, W-R Chen and F. Mallamace, Science 300, 619 (2003).

[7] F. Mallamace, C. Corsaro, H. E. Stanley, D. Mallamace, S.H. Chen. J. Chem. Phys. 139 (2013) 214502.

[8] F.H. Stillinger, J. Chem. Phys. 88 (1988) 7818-7825.

[9] J.P. Eckmann, I. Procaccia, Phys. Rev. E 78 (2008) 011503.

[10] J.C. Martinez-Garcia, J. Martinez-Garcia, S.J. Rzoska, J. Hulliger, J. Chem. Phys. 137 (2012) 064501.

[11] T. Hecksher, A.T. Nielsen, N. Boye Olsen, J.C. Dyre, Nat. Phys. 4 (2008) $737-741$.

[12] J.C. Mauro, et al., Proc. Natl. Acad. Sci. USA 106 (2009) 19780-19784.

[13] J.C. Martinez-Garcia, J.LL. Tamarit, S.J. Rzoska, J. Chem. Phys. 134 (2011) 024512. 
[14] A. Drozd-Rzoska, S.J. Rzoska, S. Pawlus, J.C. Martinez-Garcia, J.LL. Tamarit, Phys. Rev. E 82 (2010) 031501.

[15] G. Brambilla, et al., Phys. Rev. Lett. 102 (2009) 085703.

[16] L. Boue, H.G.E. Hentschel, V. Ilyin, I. Procaccia, J. Phys. Chem. B 115 (2011) 14301-14310.

${ }_{450}$ [17] G. Adams, J.H. Gibbs, J. Chem. Phys. 43 (1965) 139-146.

[18] C.A. Angell, Science 267 (1995) 1924-1935.

[19] H. Tanaka, Phys. Rev. Lett. 90 (2003) 055701.

[20] F. Mallamace, et al., Proc. Natl. Acad. Sci. USA 107 (2010) 22457.

[21] G.P. Johari, M. Goldstein, J. Chem. Phys. 53 (1970) 2372-2388.

[22] M.K. Mapes, S.F. Swallen, M.D. Ediger, J. Phys. Chem. B 100 (2006) 507.

[23] M.D. Ediger, Ann. Rev. Phys. Chem. 51 (2000) 99-128.

[24] S.F. Swallen, P.A. Bonvallet, R.J. McMahon, M.D. Ediger, Phys. Rev. Lett. 90 (2003) 015901.

[25] A. Drozd-Rzoska, S.J. Rzoska, S. Pawlus, J.LL. Tamarit, Phys. Rev. B 73 (2006) 224205.

[26] M.J. Zuriaga, S.C. Perez, L.C. Pardo, J.LL. Tamarit, J. Chem. Phys. 137 (2012) 054506.

[27] P.G. Debenedetti, F.H. Stillinger, Nature 410 (2001) 259.

[28] S.H. Chong, Phys. Rev. E 78 (2008) 041501.

${ }_{465}$ [29] F. Mallamace, C. Corsaro, H.E. Stanley, S.H. Chen, Europ. Phys. J. E 34 (2011) 11094-7.

[30] F. Stickel, E.W. Fisher, R. Richert, J. Chem. Phys. 102 (1995) 6251-6257. 
[31] S. Yip, M.P. Short, Nat. Mater. 12 (2013) 774-777.

[32] S.Corezzi, et al. J.Chem Phys. 117 (2002) 2435.

470 [33] S. Capaccioli, M. S. Thayyil and K. L. Ngai, J. Phys. Chem B 112 (2008) 16035.

[34] C.M. Roland, Soft Matter 4 (2008) 2316.

[35] S.H. Chen,et al. Proc Natl Acad Sci 103, (2006) 9012.

[36] S. Khodadadi, S. Pawlus and A. P. Sokolov, J. Phys. Chem. B 112 (2008) 14273-14280.

[37] F. Mallamace, C. Corsaro, P. Baglioni, E. Fratini and S.H. Chen J. Phys.: Condens. Matter 24 (2012) 064103.

[38] K.L. Ngai, S. Capaccioli and A. Paciaroni Chemical Physics 424 (2013) $37-44$.

[39] B.J. Berne, R. Pecora, Dynamic Light Scattering with Applications to chemistry, biology and physics. (John Wiley and Sons inc. New York, 1976).

[40] S. Rosenfeldt, et al., Angew. Chem. Int. Ed. 43 (2004) 109.

[41] N. Micali, et al., Phys. Rev. E. 58 (1998) 6229; F. Mallamace, et al., Physica A 304 (2002) 235.

[42] F. Mallamace, et al., Physica A 266 (1999) 123-135.

[43] E. Bartsch, M. Antonietti, W. Schlupp, and H. Sillescu, J. Chem. Phys.97, 3950 (1992); A. Bartsch, K. Rätzke, A. Meyer, and F. Foupel, Phys. Rev. Lett. 104, 195901 (2010)..

[44] S.H. Chong, S.H., Chen, F. Mallamace, J. Phys.: Condens. Matter 21 490 (2009) 504101.

[45] E. Rössler, K.U. Hess, V.N. Novikov, J. Non-Cryst. Solids 223 (1998) 207222. 
[46] R. Richert, C.A. Angell, J. Chem. Phys. 108 (1998) 9016-9026.

[47] L. Lobry, N. Micali, F. Mallamace, C. Liao, and S.H. Chen,Phys. Rev. E 60, 7076 (1999).

[48] W.R. Chen, S.H. Chen, and F. Mallamace, Phys. Rev. E 66, 021403 (2002); W.R. Chen, F. Mallamace, C.J. Glinka, E. Fratini, and S.H. Chen, Phys. Rev. E 68, 041402 (2003).

[49] F. Mallamace, P. Gambadauro, N. Micali, P. Tartaglia, C. Liao, and S.H. Chen, Phys. Rev. Lett. 84, 5431 (2000).

[50] F. Mallamace, P. Tartaglia, W.R. Chen, A. Faraone, and S.H. Chen, J. Phys. Condens. Matter 16, S4975 (2004).

[51] F. Mallamace, S.H. Chen, A. Coniglio, L. de Arcangelis, E. Del Gado, and A. Fierro, Phys. Rev. E. 73, 020402 (2006).

[52] S. Caponi, et al., Phys. Rev. B 79 (2009) 172201.

[53] D. Prevosto, et al., J. Non-Cryst. Solids. 355 (2009) 705-711.

[54] R. Böhmer, et al., Phys. Rev. Lett. 97 (2006) 135701.

[55] S.P. Das, G.F. Mazenko, Phys. Rev. A 34 (1986) 2265-2282.

[56] L. Berthier, W. Kob, J. Phys.: Condens. Matter 19 (2007) 205130.

[57] G. Szamel, E. Flenner, Europhys. Lett. 67 (2004) 779-785.

[58] R.F. Weysser, M. Fuchs, Phys. Rev. Lett. 105 (2010) 169604.

[59] W. van Megen, V.A. Martinez, G. Btyant, Phys. Rev. Lett. 102 (2009) 168301.

[60] M. Goldstein, J. Chem. Phys. 132 (2010) 041104. 


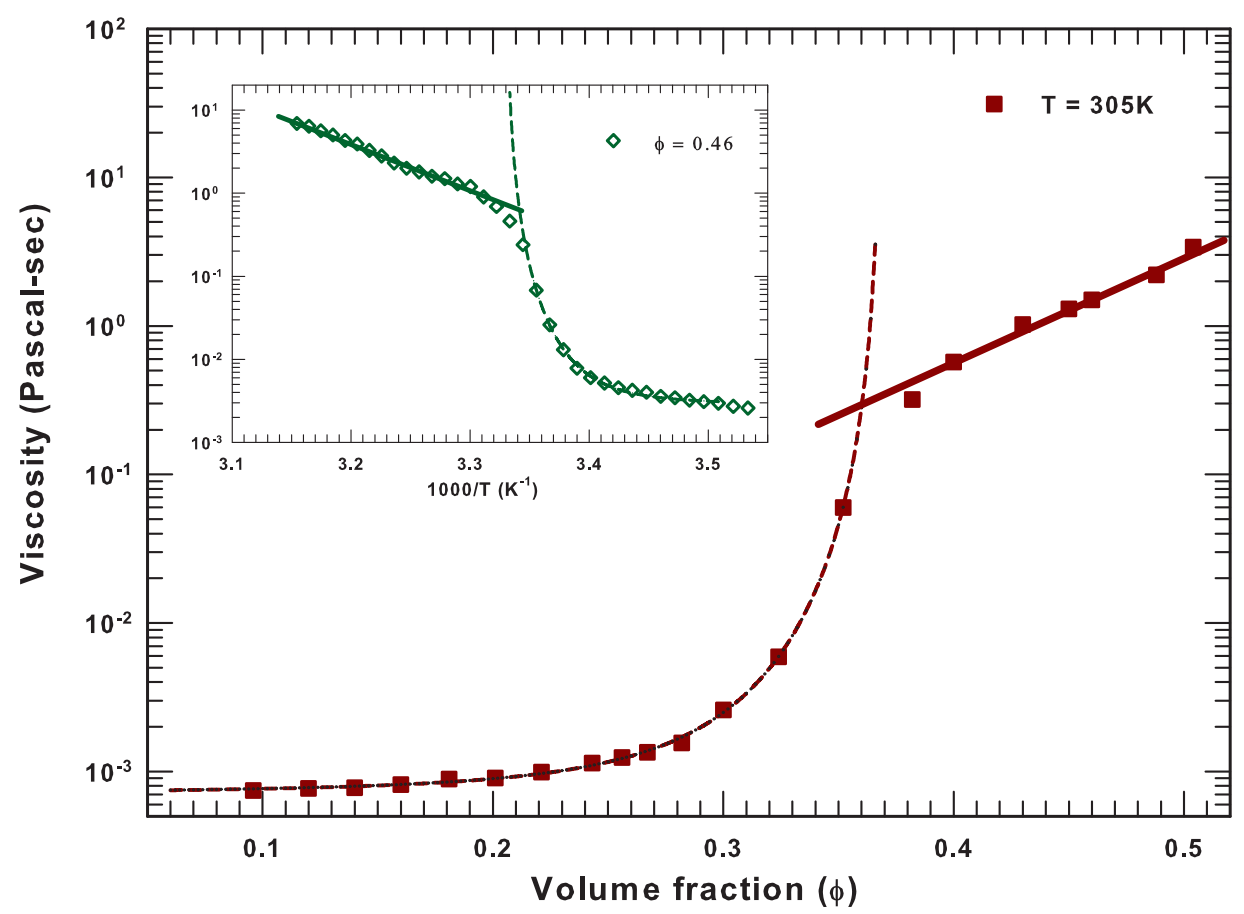

Figure 1: The viscosity of the $P L 64 / D_{2} O$ AHS micellar system measured as a function of the volume fraction at $\mathrm{T}=305 \mathrm{~K}$ is reported in a log-lin scale. The data are fitted at low $\phi$ by using the MCT power law (Eq.3) with $\phi$ as the control parameter (short dashed line). The dynamical crossover from a strong to fragile glass former behavior is observable at $\phi_{c} \simeq 0.37$. In the inset, the log-lin plot of the viscosity of the same micellar system at the volume fraction of 0.46 is reported. The short dashed line represents the data fitting at low $\mathrm{T}$ in terms of the MCT power law (Eq.3) with $\mathrm{T}$ as the control parameter. At this volume fraction the dynamical crossover from super-Arrhenius to Arrhenius behavior (continuous line) can be easily observed. The obtained crossover temperature $T_{c}$ is about $300 \mathrm{~K}$. 


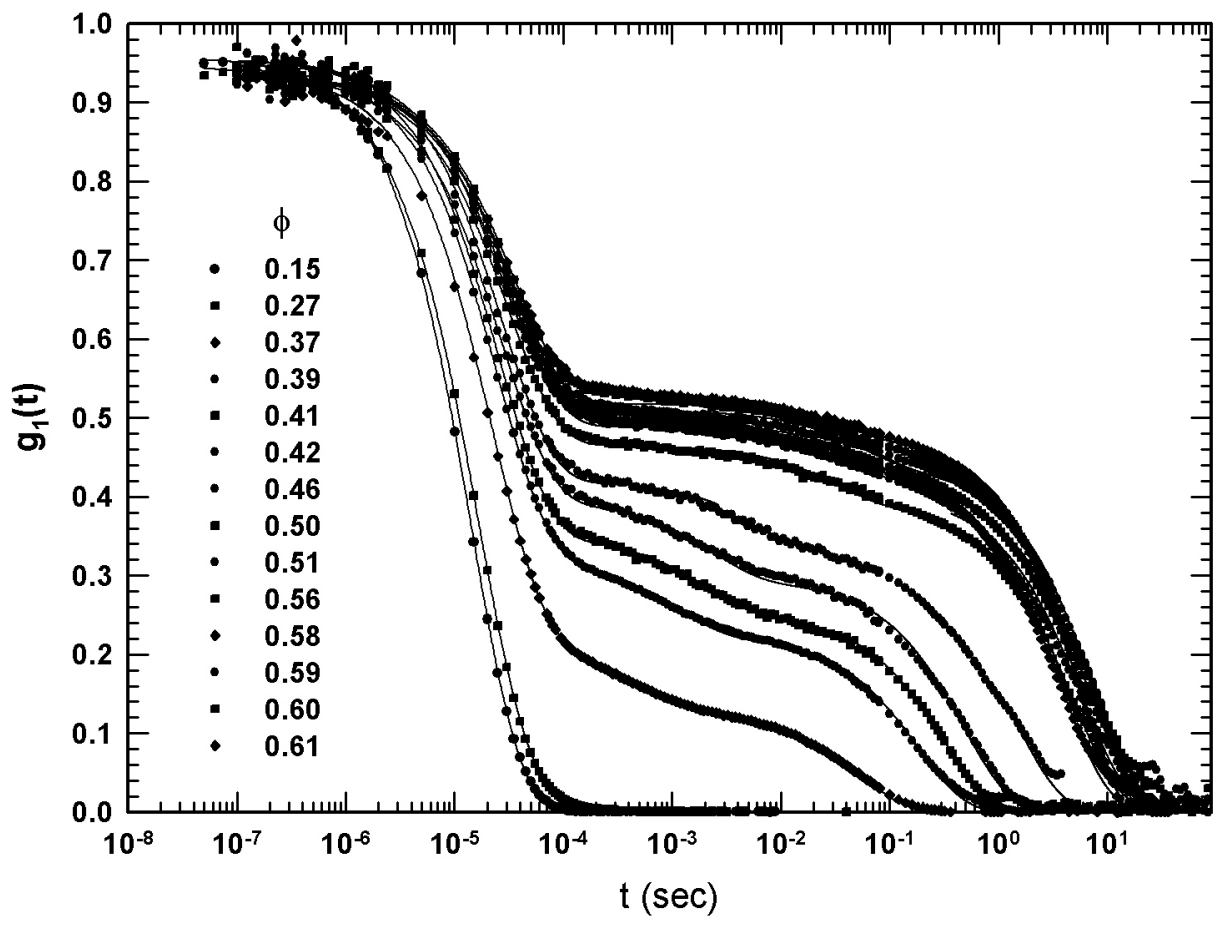

Figure 2: The correlator $g_{1}(t)$ for different values of the volume fraction for PAMAM dendrimer in methanol. The continuous lines represent the best fit obtained by the superposition of an exponential short-time decay (the $\beta$-fast contribution) and two stretched tails weighted for the non-ergodicity factor $f_{c}$. 


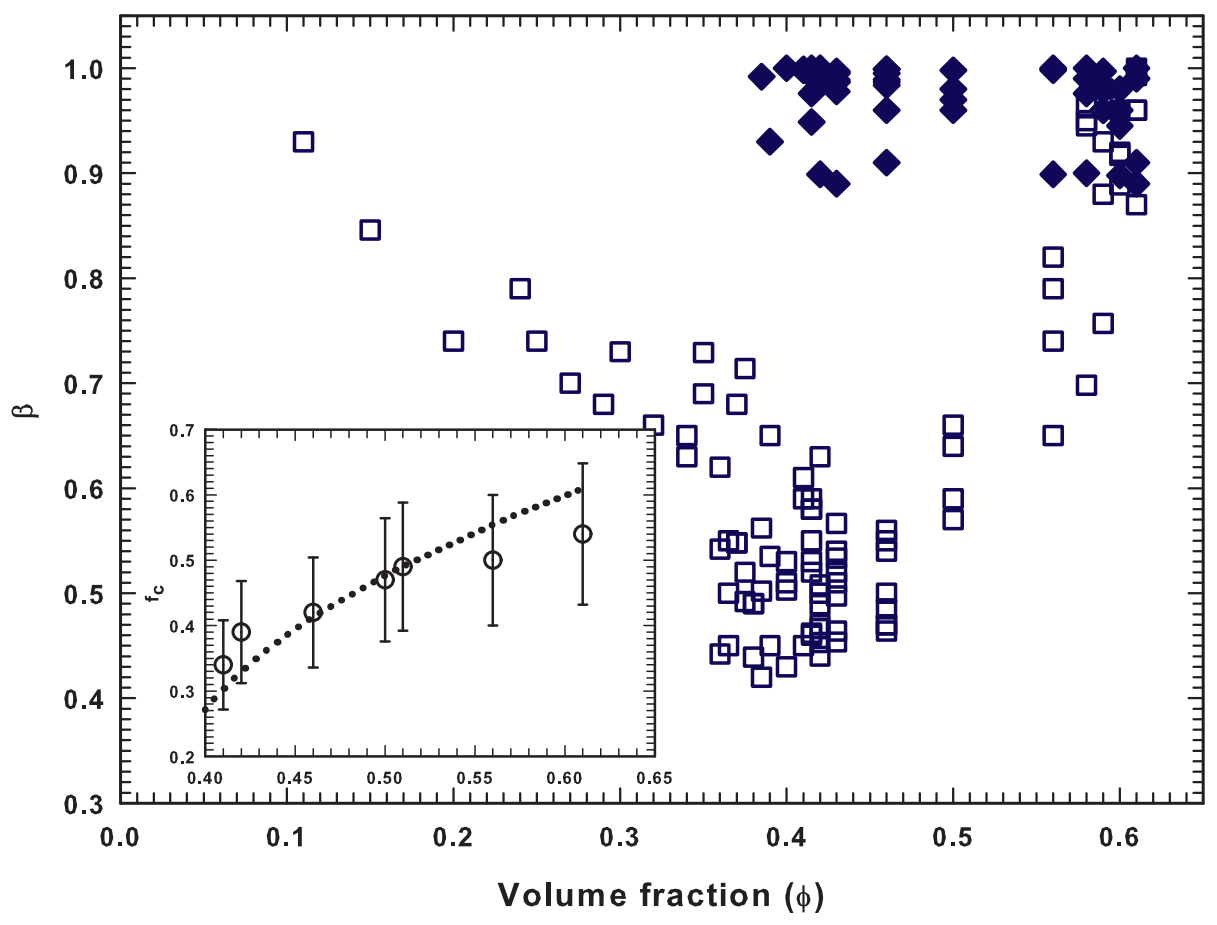

Figure 3: The non-ergodicity factor $f_{c}$ (inset) and the stretched exponent $\beta$ obtained from the measured correlators of PAMAM dendrimers in methanol. $\beta_{\text {inter. }}$ data are reported as closed symbols and the $\beta$ values as open symbols. The Debye-Waller factor $f_{c}$ is reported for different volume fractions in the range 0.4-0.65. The dotted line represents the MCT scaling law prediction, as the volume fraction increases above $\phi_{c}: f_{c} \simeq\left|\phi_{c}-\phi\right|^{1 / 2}$. 


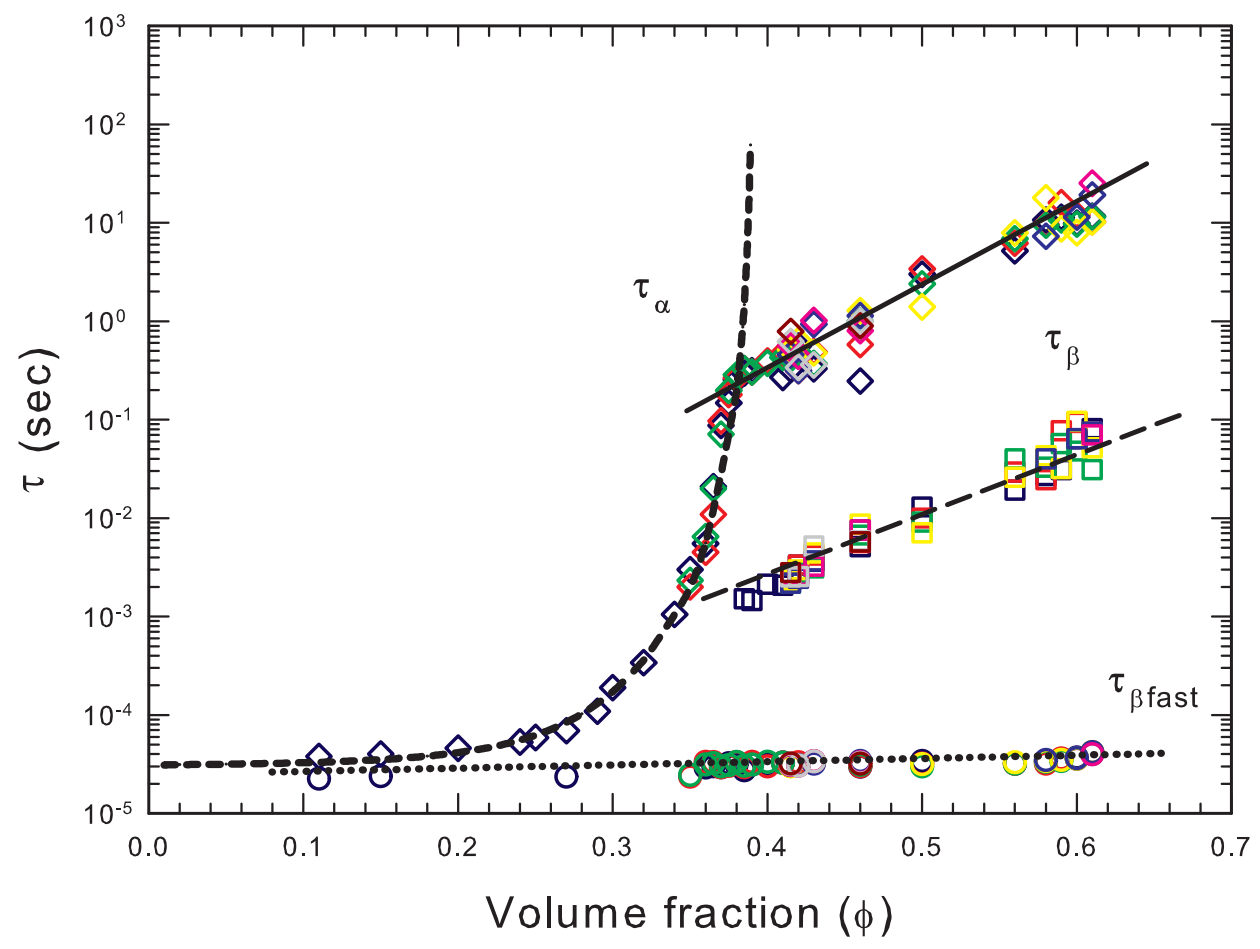

Figure 4: The relaxation times here measured in the range $0.11<\phi<0.61$ show that a bifurcation from the $\alpha$ process occurs close to $\phi_{c}$ (at the relaxation time of about $0.2 \mathrm{sec}$ ), where $\tau_{\beta}$ has its onset with an Arrhenius T-dependence. 